\title{
The Compact Genetic Algorithm for Likelihood Estimator of First Order Moving Average Model
}

\author{
Rawaa Dawoud Al-Dabbagh ${ }^{1}$, Dr. Mohd. Sapiyan Baba ${ }^{1}$, Dr. Saad Mekhilef ${ }^{2}$, Azeddien Kinsheel ${ }^{3}$ \\ ${ }^{1}$ Department of Artificial Intelligence \\ ${ }^{2}$ Department of Electrical Engineering \\ ${ }^{3}$ Department of Engineering Design and Manufacturing \\ University of Malaya \\ Kuala Lumpur, Malaysia \\ rawaa_aldabbagh@siswa.um.edu.my, pian@um.edu.my, saad@um.edu.my, azkensh@gmail.com
}

\begin{abstract}
Recently Genetic Algorithms (GAs) have frequently been used for optimizing the solution of estimation problems. One of the main advantages of using these techniques is that they require no knowledge or gradient information about the response surface. The poor behavior of genetic algorithms in some problems, sometimes attributed to design operators, has led to the development of other types of algorithms. One such class of these algorithms is compact Genetic Algorithm (cGA), it dramatically reduces the number of bits reqyuired to store the poulation and has a faster convergence speed. In this paper compact Genetic Algorithm is used to optimize the maximum likelihood estimator of the first order moving avergae model $M A(1)$. Simulation results based on MSE were compared with those obtained from the moments method and showed that the Canonical GA and compact GA can give good estimator of $\widehat{\boldsymbol{\theta}}$ for the $M A(1)$ model. Another comparison has been conducted to show that the cGA method has less number of function evaluations, minimum searched space percentage, faster convergence speed and has a higher optimal precision than that of the Canonical GA.
\end{abstract}

Keywords-Moving Average (MA), Likelihood Function, Moment Estimation Method, Canonical Genetic Algorithm (CGA), compact Genetic Algorithm (cGA), Mean Square Error (MSE).

\section{INTRODUCTION}

One of the most famous procedures for the solution of optimization problems is Genetic Algorithms (GAs). GA is composed mainly of three steps: recombination, crossover and mutation. By maintaining a population of solutions, GA can be viewed as implicitly modeling of the solutions seen in the search process. In the standard GA, new solutions are generated by applying randomized recombination operators on two or more high-quality individuals of the current population [1]. These recombination operators, such as onepoint, two-point or uniform crossover, randomly selected non- overlapping subsets of two "parent" solutions to form "children" solutions.
The poor behavior of genetic algorithms in some problems, sometimes attributed to designed operators, has led to the development of other types of algorithms such as the Probabilistic Model Building Genetic Algorithms (PMBGAs) or Estimation of Distribution Algorithms (EDAs). They are a class of algorithms which have been developed recently to preserve the building blocks [2]. The principal concept in these new techniques is to prevent the distribution of partial solutions contained in a solution by building a probabilistic model [2] [3] [4]. To name just a few, instances of EDA algorithms include the Populationbased Incremental Learning (PBIL) [5] [6] and the compact Genetic Algorithm (cGA) [7]. The compact GA represents the population as a probability (distribution) vector $(P V)$ over the set of solutions and is operationally equivalent to the order-one behavior of the simple GA with uniform crossover. It processes each gene independently and requires less memory than simple GA [7] [2]. As a case study to investigate the relative performance of cGA for optimizing the solution of estimation problem, we have utilized cGA for optimizing the maximum likelihood $\ln \left(L\left(\theta, \sigma_{a}^{2}\right)\right)$ function of the first order moving average model $M A(1)$.

A time series is an ordered sequence of observations in an equal interval space; this ordering is generated through time or other dimensions such as sapce. Time series occur in a variety of fields (such as engineering, economics and agriculture). As one of the distinguished stochastic models which represent time series is the simple moving average and it is also called a first order moving average, denoted by $M A(1)$ because it contains just one parameter, $\theta$. A generalization of the $M A(1)$ model is the $q^{\text {th }}$ order moving average which is denoted by $M A(q)$ and takes the formula [8]

$$
\begin{gathered}
z_{t}=a_{t}-\theta_{1} a_{t-1}-\cdots-\theta_{q} a_{t-q} \\
z_{t}=\theta(B) a_{t} \\
\theta(B)=1-\theta_{1} B-\cdots-\theta_{q} B^{q-1}
\end{gathered}
$$

where $a_{t} \sim$ idd $N\left(0, \sigma_{a}^{2}\right)$, meaning that the $a_{t}$ are identically, independently distributed, each with a normal distribution having mean 0 and the same variance. This model employes $q+1$ unknown parameters $\theta_{1}, \theta_{2}, \ldots, \theta_{q}, \sigma_{a}^{2}$ that are estimated from the data. The moving average 
process is stationary for any value of $\theta_{1}, \theta_{2}, \ldots, \theta_{q}$, that is, the mean and the variance of the underlying process are constant and auto-covariance's depends only on the time lag. But many economic and business time series are somtimes considered to be non-stationary. Non-stationary time series can occur in many different ways. Sometimes the series has a non-stationary behavior about a fix mean, and hence its behavior can be represented by a model which calls for $d^{\text {th }}$ difference of the process to be stationary. In practice $d$ is usually 0,1 , or at most 2 . The difference provides a powerful model for describing stationary and non-stationary time series and it is called an integrated moving average (IMA) process or ARIMA of order $(0, d, q)$. The process which is defined by first order, is known as $\operatorname{ARMA}(0, d, q)$, it takes the formula

$$
w_{t}=a_{t}-\theta_{1} a_{t-1}
$$

or in its operator form:

$$
w_{t}=\theta(B) a_{t}
$$

where $w_{t}=\nabla^{d} z_{t}$ and the moving average operator $\theta(B)=$ $1+\theta_{1} B+\theta_{2} B^{2}+\cdots+\theta_{q} B^{q}$, here the moving average process is stationary for any values of $\theta_{1}, \theta_{2}, \ldots, \theta_{q}$.

This model will be invertible if the root of $\left(1-\theta_{1} B=0\right)$ lies outside the unit circle; an inertible $M A$ is time reversible, so we can get that $\left|\theta_{1}\right|<1$. Moments of this stationary model are:

Mean Function

Variance Function

$$
E(Z)=0
$$

$$
\gamma_{0}=\operatorname{var}\left(z_{t}\right)=\left(1+\theta_{1}^{2}\right) \sigma_{a}^{2}
$$

Auto-Covariance Function

$$
\gamma_{k}=\left[\begin{array}{cc}
-\theta_{1} \sigma_{a}^{2} & k=1 \\
0 & k>1
\end{array}\right]
$$

Auto-Correlation Function

$$
\rho_{k}=\left[\begin{array}{cc}
1 & k=0 \\
\frac{-\theta_{1}}{1+\theta_{1}^{2}} & k=1 \\
0 & \text { e.w }
\end{array}\right]
$$

We can show that the marginal distribution of time series that has $M A(1)$ model is $z_{t} \sim N\left(0,\left(1+\theta_{1}^{2}\right) \sigma_{a}^{2}\right)$ if $a_{t} \sim N\left(0, \sigma_{a}^{2}\right)$. Estimation is the second step in analysis of the time series. It indicates an effecient use of the data to make inferences about the parameters conditional to the adequacy of the entrtained model. ARMA models can be difficult to estimate if the parameter estimates are not within the appropriate range, a moving average model's residual terms will grow exponentially. The calculated residuals for later observations can be very large or can overflow. This can happen either because of improper starting values being used or because the iterations moved away from reasonable values. Moreover, our model is nonlinear because $a_{t}=$ $\frac{1}{1-\theta_{1} B}$, so there is no direct method that can handle these limitations, but all suitable methods are indirect methods
(Iterative method) which start with an initial value and then this value is modified iteratively by using some numerical algorithms. The numerical method gives an approximate estimator with some accuracy.

Recently, B. Hussain [9] [10] proposed the use of Canonical Genetic Algorithm (CGA) for optimizing the maximum likelihood function $\ln \left(L\left(\theta, \sigma_{a}^{2}\right)\right)$ of the first order moving average $M A(1)$ model. And its results were compared with the results obtained by the moment estimator method.

In this paper we introduce a new evolutionary way to estimate the same model by using the compact Genetic Algorithm (cGA). Droste [11] proofed that cGA is applicable to be used for optimizing most of the linear functions such One-Max in the optimal expected runtime. In this paper we are interested in the behavior of the cGA for non-linear functions in comparison with CGA according to the number of function evaluations taken, solution quality, the percentage of the search space searched until convergence and the convergence speed.

The rest of the paper is organized as follows. Section 2 explains the problem formulation of maximum likelihood estimator. Section 3 briefly describes the standard cGA. Section 4 presents the simulation results obtained from applying CGA, cGA and moment method for optimizing the model under study. Section 6 concludes the paper.

\section{PROBLEM FORMULATION}

Maximum likelihood estimator (MLE) is a standard approach to parameter estimation and inference in statistics; it is a method that finds the most likely value for the parameter based on the data set collected, in particular in non-linear modeling with non-normal data. MLE has many optimal properties in estimation: sufficiency (complete information about the parameter of interest contained in its MLE estimator); consistency (true parameter value that generated the data recovered asymptotically, i.e. for data of sufficiently large samples); efficiency (lowest-possible variance of parameter estimates achieved asymptotically); and parameterization invariance (same MLE solution obtained independent of the parameterization used) [12].

Assume that a time series which is denoted by $z_{-d+1}, \ldots, z_{0}, z_{1}, z_{2}, \ldots, z_{n}$ is generated by an $\operatorname{IMA}(1,1)$ model over $N=n+d$ original observations $z$. From these observations, a series $w=\left\{w_{1}, w_{2}, \ldots, w_{n}\right\}$ of $n=N-d$ is generated where $w_{t}=\nabla^{d} z_{t}$ and $n$ is the sample size. The stationary mixed $\operatorname{ARMA}(0,1)$ model in Eq.7 is rewritten as [13]

$$
a_{t}=w_{t}+\theta_{1} a_{t-1}
$$

where $E\left(w_{t}\right)=0$. Suppose that $\left\{a_{t}\right\}$ has the normal ditribution with zero mean and constant variance equal to $\sigma_{a}^{2}$ , then the likelihood function can be written as follows [13]:

$$
L=\left(2 \pi \sigma^{2}\right)^{-\frac{n}{2}}\left|M^{(0,1)}\right|^{\frac{1}{2}} \exp \left(\frac{-S\left(\theta_{1}\right)}{2 \sigma_{a}^{2}}\right)
$$

The logarithmic likelihood function is given by:

$$
\operatorname{Ln}(L)=-\frac{n}{2}\left(2 \pi \sigma_{a}\right)+\frac{1}{2} \ln \left(1-\theta_{1}^{2}\right)-\frac{s\left(\theta_{1}\right)}{2 \sigma_{a}^{2}}
$$


where

$$
S\left(\theta_{1}\right)=\sum_{t=1}^{n} a_{t}^{2}\left(\theta_{1} \mid z_{0}, \theta_{1}, z\right)
$$

is the sum squares errors and $\left[a_{t} \mid \theta_{1}, w\right]=E\left[a_{t} \mid \theta_{1}, w\right]$ denotes the expectation of $a_{t}$ conditional on $\theta_{1}$ and $w$. The sum squares of errors can be found by unconditional calculation of the $[a]$ 's which are computed recursively by taking expectations in Eq.12, it is also called Least Square Estimate (LSE) in which the parameter estimated is obtained my minimizing the sum of square in Eq.12, it usually provides very close approximation to the maximum likelihood estimator. Back-forecasting is a popular technique, it estimates the parameters which are crudely put into the model and run backwards in time; a back-calculation provides the values $\left[w_{-j}\right], j=0,1,2, \ldots$ this back-forecasting is needed to start off the forward recursion. For moderate and large values of $n$, Eq. 12 is dominated by $S\left(\theta_{1}\right) / 2 \sigma_{a}^{2}$ and thus the contours of the unconditional sum squares function in the space of the parameters $\theta$ are very nearly contours of likelihood and log-likelihood.

For a causal and finite auto-regressive equation, the method of moment estimators according to the Yule-Walker equations almost coincides with the least squares or maximum Gaussian likelihood estimators and their consistency as well as their asymptotic normality may be established. The standard Yule-Walker equations, as they are known for an auto-regression, are generalized to involve the moments of a moving-average process indexed on any number of dimensions [14]. It is obtained by equating a sample moment such as sample mean $\bar{z}$, sample variance $\widehat{\gamma}_{0}$, and sample ACF (Auto-correlation Function) $\hat{\rho}_{i}$ to their theoretical values counterparts and solving the resultant equations.

For the presented model, the estimate of parameters can be obtained by equating sample moments to their theoretical values. Approximate values for the parameters are obtained by substituting the estimators $r_{k}$ for $\rho_{k}$.

$$
\hat{\rho}_{k}=r_{k}=\frac{\sum_{t=1}^{n-k}\left(z_{t}-\bar{z}\right)\left(z_{t+k}-\bar{z}\right)}{\sum_{t=1}^{n}\left(z_{t}-\bar{z}\right)}
$$

Hence, from Eq.8 and the correlation function $\rho=\frac{-\theta}{1+\theta_{1}}$ we get [13]:

and

$$
\begin{gathered}
\hat{\rho}_{1}=r_{1}=\frac{-\widehat{\theta}_{1}}{1+\widehat{\theta}_{1}^{2}} \\
\hat{\theta}_{1}=\frac{-1 \pm \sqrt{1-4 r_{1}^{2}}}{2 r_{1}}
\end{gathered}
$$

where $\hat{\theta}_{1}$ is the value that makes $\sqrt{1-4 r_{1}^{2}}>0$ and $|\hat{\theta}|<1$.

\section{THE COMPACT GENETIC ALGORITHM (cGA)}

The compact Genetic Algorithm (cGA) is similar to the PBIL (population Based Incremental Learning) but requires fewer steps, fewer parameters and less of a gene sample [11].

The cGA manages its population as a probability vector $(P V)$ over the set of solutions (i.e., only models its existence), therby mimicking the order-one behavior of the sGA with uniform crossover using a small amount of memory [1] [15].
Parameters. $\quad n$ : population size $\quad l$ : chromosome length

Step1. Initialize probability vector

For $i:=1$ to $l$ do $p[i]:=0.5$;

Step2. Generate two chromosomes from the probability vector $a:=\operatorname{generate}(p) ; \quad b:=\operatorname{generate}(p)$

Step3. Let them compete

winner, loser := compete $(a, b)$;

Step4. Update the probability vector

$$
\begin{aligned}
& \text { for } i:=1 \text { to } l d o \\
& \text { if winner }[i] \neq \operatorname{loser}[i] \text { then } \\
& \text { if winner }[i]==1 \text { then } \\
& p[i]:=p[i]+1 / n \\
& \text { else } p[i]:=p[i]-1 / n \text {; }
\end{aligned}
$$

Step5. Check if the probability vector has converged. Go to Step 2 , if it is not satisfied.

Step6. The probability vector $p[i]$ represents the final solution.

Figure 1. Pseudo code fashion of the cGA

Figure 1 and 2 describes the pseudo-code and flowchart of the cGA. The values of $P V p_{i} \in[0,1], \forall_{i}=1, \ldots, l$, where $l$ is the number of genes (i.e., the length of the chromosome), measures the proportion of " 1 " alleles in the $i^{\text {th }}$ locus of the simulated population [2][15]. The $P V$ is initially assigned 0.5 to represent a randomly generated population. In every generation (i.e., iteration), competing chromosomes are generated on the basis of the current $P V$, and their probabilities are updated to favor a better chromosome (i.e, winner). It is noted that the generation of chromosomes from $P V$ simulates the effects of crossover that leads to a decorrelation of the population's genes. In a simulated population of size $n$, the probability $p_{i}$ is increased (decreased) by $1 / n$ when the $i^{t h}$ locus of the winner has an allel of " 0 "(" 1 "). If both the winner and the loser have the same allele in each locus, then the probability remains the same. This scheme is equivalent to (steady-state) pair-wise tournament selection [1]. The cGA is terminated when all the probabilities converge to zero or one. The convergent $P V$ itself represents the final solution. It seen that the cGA requires $l \times \log _{2}(n+1)$ bits of memory while the sGA requires $l \times n$ bits [1]. Thus, large population size can be effectively exploited without unduly compromising on memory requirements [15].

\section{A. Individual Initialization and Encoding:}

Certain restrictions are defined on the encoding scheme:

1. cGA needs in every step two random numbers, each having a bit-string (0's and 1's) of fixed length $l=15$ bits. Two individuals $a$ and $b$ are generated: they are two identical chromosomes working in parallel, but using different initial seeds. Each individual affectionately known as a critter represents an element with the domain of the 
solution space of the optimization problem. The chromosome of a given critter is the only source for of all the information about the corresponding solution. To apply the cGA to real-values parameters optimization problems of the form $f: \Pi\left[u_{i}, v_{i}\right] \rightarrow R\left(u_{i}<v_{i}\right)$, the bit-strings is logically divided into $n$ segments of (in most cases) equal length $l_{x}\left(l=n l_{x}\right)$ and each segment is interpreted as the binary code of the corresponding object variable $x_{i} \in\left[u_{i}, v_{i}\right]$. A segment decoding function $\Gamma^{i}:\{0,1\}^{l_{x}} \rightarrow\left[u_{i}, v_{i}\right]$ typically looks like

$\Gamma^{i}\left(a_{i 1} a_{i 2} \ldots a_{i l_{x}}\right)=u_{i}+\frac{v_{i}-u_{i}}{2^{l} x-1}\left[\sum a_{i j} 2^{j-1}\right]$,

where $\left(\begin{array}{llll}a_{i 1} & a_{i 2} & \ldots & a_{i l_{x}}\end{array}\right)$ denotes the $i^{t h}$-segment of an individual $\vec{a}=\left(a_{11}, \ldots, a_{n l_{x}}\right) \in l^{n l_{x}}=I^{l}$.

2. Every field of the probability vector $P V$ is nitialized to 0.5 .

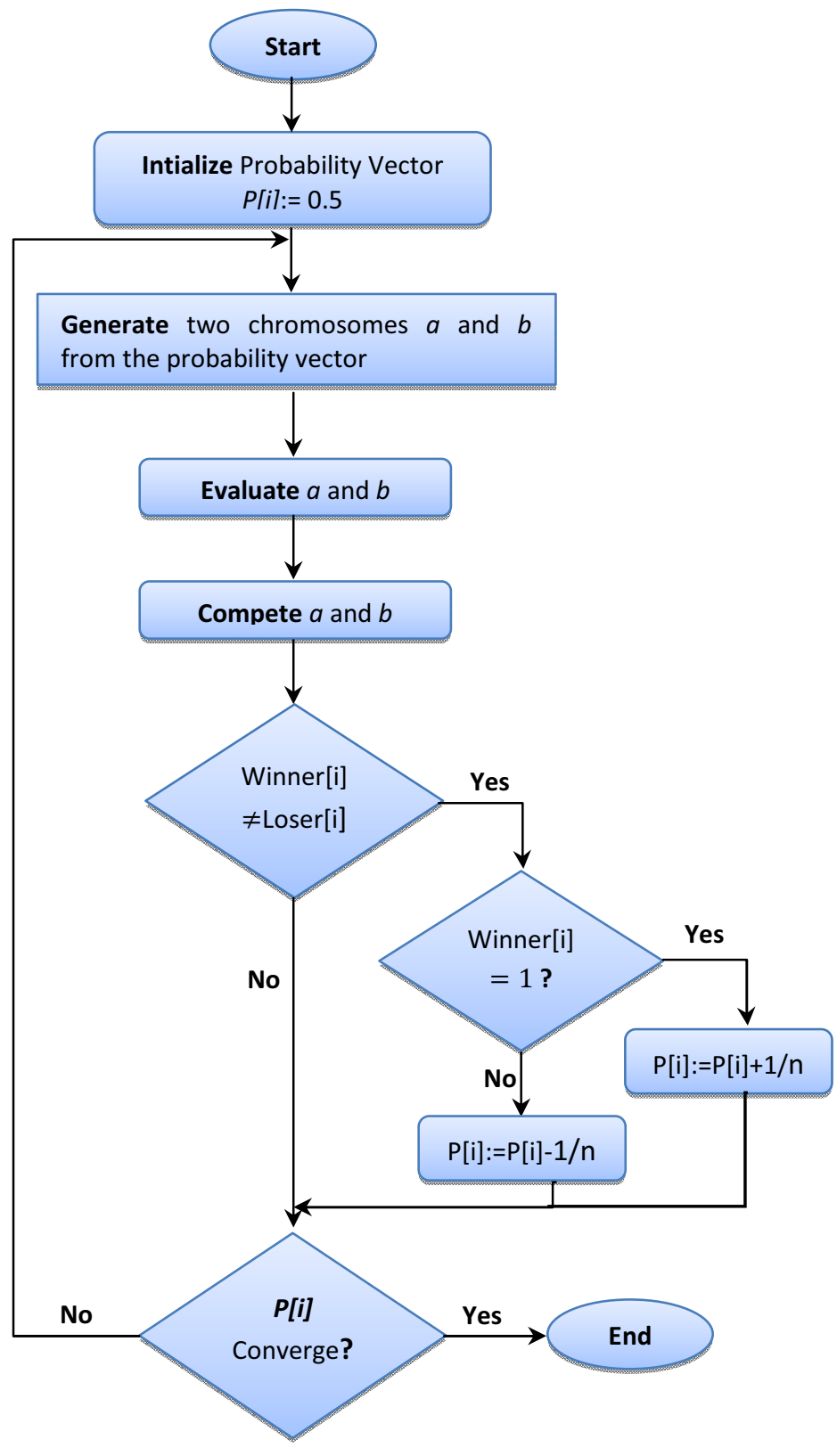

Figure 2. Flowchart of cGA

\section{B. Fitness Evaluation:}

A fitness function is a numerical value associated with each individual to measure the goodness of the solution. Each individual $a$ and $b$ is converted into a number between 0 and 32768; $\left\{15\right.$ bits mean $2^{15}$ possible values $\}$. So, individual with higher fitness value represents better solution, while lower fitness value is attributed to the individual whose bitstring represents inferior solution. Combinig the segmentwise decoding function to individual-decoding function $\Gamma=$ $\Gamma^{1} \times \ldots \times \Gamma^{n},[16]$ fitness values are obtained by setting

$$
\Phi(\vec{a})=\delta(f(\Gamma(\vec{a})))
$$

where $\delta$ denotes a scaling function ensuring positive fitness values such that the best individual receives largest fitness.

\section{Compete:}

Compete is a procedure that compares 2 real-values (meaning 2 bit-strings), $a$ and $b$ and has an output either ' 1 ' (if $a>b$ ), or ' 0 ' (if $a<b$ ). The comparison depends on the Fitness Evaluation module.

\section{Probability Update}

As the population has $n$ chromosomes, the probability vector $P V$ must be able to be increased or decreased by a minimal value of $1 / n$. There is no need to represent the probability as the float number actually is.

As the probability has always values between ' 0 ' and ' 1 ' and can be written as the sum of the negative powers of 2 , with ' 0 ' or ' 1 ' as coefficients, the probability vector contains the bit-string of these coefficients. Increasing and decreasing it by the minimal value means to change at least one value of this bit-string. Technically, the $p[i]$ is updated as follows:

$$
\begin{aligned}
& \text { if } f_{a} \geq f_{b} \text { then } \\
& \qquad \begin{array}{l}
\text { if } a[i]=1 \text { then } p[i]=\min \left(1, p[i]+\frac{1}{n}\right) \\
\text { if } a[i]=0 \text { then } p[i]=\max \left(0, p[i]-\frac{1}{n}\right)
\end{array}
\end{aligned}
$$

else

$$
\begin{aligned}
& \text { if } b[i]=1 \text { then } p[i]=\min \left(1, p[i]+\frac{1}{n}\right) \\
& \text { if } b[i]=0 \text { then } p[i]=\max \left(0, p[i]-\frac{1}{n}\right)
\end{aligned}
$$

So, the probability vector $P V(p[i])$ stores the bit-string that represents the probability. The operations that it needs to perform are increased and decreased the bit-string by one unit.

\section{RESULTS AND DISCUSSION}

This section presents simulation results and compares the compact GA and Canonical GA in terms of solution quality, the number of function evaluations and the percentage of the searched space taken for the likelihood estimator of $M A(1)$. Furthermore, the results of these former methods have been 
compared with those obtained by simulating the moment method with 1000 runs [9] [10]. The initial population genes were randomly assigned with values within the range $[-1.0$, 1.0]. The simulation results performed are based on different sample size (i.e. $n=25,50,100), \theta$ is set to $(\mp 0.1, \mp 0.4, \mp 0.7)$. The random variables $a_{t}{ }^{\prime}$ s are generated by using Box-Muller formula and sample of size $n$ generated by Eq. 3. The comparison has been based on Mean Square Error $M S E=\operatorname{var}(\theta)+$ bias. All simulations are averaged over 100 runs.

The canonical GA used binary tournament selection without replacement, and uniform crossover with exchange probability $P_{c}=0.75$. Inversion mutation is used with probability $P_{m}=0.005$. The size of population is set to 50 . All runs end when the population fully converged that is when the individuals have the same alleles at each gene position. Putting this all together we obtain a CGA as summarized in Table 1.

Table 1: Tableau describing the CGA for the likelihood estimator

\begin{tabular}{|l|l|}
\hline Representation & Binary bit string of length $l$ \\
\hline Recombination & Two point crossover \\
\hline $\begin{array}{l}\text { Recombination } \\
\text { Probability }\end{array}$ & $75 \%$ \\
\hline Mutation & $\begin{array}{l}\text { Each value inverted with } \\
\text { independent probability } P_{m} \text { per } \\
\text { position }\end{array}$ \\
\hline Mutation Probability & $0.005 \%$ \\
\hline Parent Selection & $\begin{array}{l}\text { Tournament selection (best out } \\
\text { of two) }\end{array}$ \\
\hline Population size & 50 \\
\hline Number of off spring & 50 \\
\hline Initialization & Random \\
\hline $\begin{array}{l}\text { Termination } \\
\text { Condition }\end{array}$ & $\begin{array}{l}\text { No improvement in the last } 10 \\
\text { generations }\end{array}$ \\
\hline
\end{tabular}

As opposed to CGA, in compact GA the population size $P_{s}$ and the chromosome length $l$ are set to $30-50$ and 15 respectively. The algorithms start with a probability register initialized with 0.5 , so that at the beginning, there are equal chances for every bit of the future chromosome to be either' 0 ' or ' 1 ' at the end of the algorithm. The objective function decides whether it is better to increase or decrease the entry in the probability register. Table 2 illustrates the results and the simulations on a set of data that gives some ideas of the behavior of compact GA, Bitweight cGA, Canonical GA and Moment Method.

From Table 2 we can see that the MSEs of cGA, and CGA are relatively competing in a small range of differences but they are all smaller than those obtained from the moment estimator. Consequently, they are more reliable than the moment method in estimating the parameters of the model under study. On the other hand, the value of the MSE decreases when the sample size increases for all the adopted methods. Moreover, from the behavior of MSE of cGA and CGA it can be discerned that when the model parameters $\theta_{1}$ take positive values is smaller than when these parameters are assigned to a negative ones.

Table 3 illustrates the average simulation results of Canonical GA and compact GA respectively with population size $\left(P_{S}=50\right)$ over 100 runs, where ( $F$ is the number of function evaluations taken until convergence for the various numbers of generations) and (PSS is the percentage \% of the searched space) and it is calculated as follows:

$$
P S S=\frac{N C \times N G}{T S S} \times 100
$$

where

$N C=$ number of individuals being evaluated per generation

$N G=$ number of generations until convergence

$T S S=$ total search space size

and (total search space size $\left.=2^{l}\right)$

(no. of chrom. being evaluated $=P_{s}$ in CGA and 2 in compact GA)

Table 2: MSE for Canonical GA, compact GA and moment method for different values of sample size and model parameter.

\begin{tabular}{|c|c|c|c|c|c|c|}
\hline \multirow[t]{2}{*}{$\begin{array}{l}\text { Sample } \\
\text { Size (n) }\end{array}$} & \multirow[t]{2}{*}{$\theta_{1}$} & \multirow{2}{*}{$\begin{array}{l}\text { MSE of } \\
\text { moment } \\
\text { estimator }\end{array}$} & \multicolumn{2}{|c|}{$\begin{array}{c}\text { MSE of } C G A \\
\text { estimator }\end{array}$} & \multicolumn{2}{|c|}{$\begin{array}{c}\text { MSE of cGA } \\
\text { estimator }\end{array}$} \\
\hline & & & $\begin{array}{c}\text { For } \\
\text { best } \theta_{1}\end{array}$ & $\begin{array}{c}\text { For } \\
\text { average } \\
\theta_{1}\end{array}$ & $\begin{array}{c}\text { For } \\
\text { best } \\
\theta_{1} \\
\end{array}$ & $\begin{array}{c}\text { For } \\
\text { average } \\
\theta_{1}\end{array}$ \\
\hline \multirow{6}{*}{25} & 0.1 & 0.4353 & 0.2866 & 0.2235 & 0.3001 & 0.255 \\
\hline & -0.1 & 0.6743 & 0.8637 & 0.513 & 0.738 & 0.5021 \\
\hline & 0.4 & 0.5602 & 0.1448 & 0.06113 & 0.1365 & 0.0563 \\
\hline & -0.4 & 0.7405 & 0.40122 & 0.3634 & 0.334 & 0.3874 \\
\hline & 0.7 & 1.1845 & 0.2331 & 0.1181 & 0.229 & 0.1213 \\
\hline & -0.7 & 1.3421 & 0.4197 & 0.36502 & 0.4201 & 0.3771 \\
\hline \multirow{6}{*}{50} & 0.1 & 0.2163 & 0.1872 & 0.2144 & 0.2163 & 0.2182 \\
\hline & -0.1 & 0.3421 & 0.357 & 0.2905 & 0.3412 & 0.3301 \\
\hline & 0.4 & 0.50602 & 0.0785 & 0.02353 & 0.0843 & 0.0283 \\
\hline & -0.4 & 0.4765 & 0.3231 & 0.20019 & 0.3415 & 0.19202 \\
\hline & 0.7 & 1.0845 & 0.2056 & 0.1004 & 0.1987 & 0.1045 \\
\hline & -0.7 & 1.1233 & 0.2543 & 0.29902 & 0.2455 & 0.2712 \\
\hline \multirow{6}{*}{100} & 0.1 & 0.0799 & 0.01334 & 0.0763 & 0.0224 & 0.0654 \\
\hline & -0.1 & 0.1038 & 0.2705 & 0.1225 & 0.2425 & 0.1446 \\
\hline & 0.4 & 0.3602 & 0.0145 & 0.0122 & 0.0133 & 0.0113 \\
\hline & -0.4 & 0.3212 & 0.2806 & 0.0351 & 0.2543 & 0.0344 \\
\hline & 0.7 & 1.01845 & 0.1232 & 0.0677 & 0.1336 & 0.0745 \\
\hline & -0.7 & 0.9999 & 0.1408 & 0.0123 & 0.1581 & 0.0168 \\
\hline
\end{tabular}

Formally speaking, there is an evidence that the two algorithms are quite different, while CGA has a memory requirement of $\left(P_{S} \times \mathrm{l}\right)$ bits, the cGA requires only $\left(\log _{2} P_{s} \times l\right)$ bits and in the number of function evaluations CGA requires $\left(P_{S} \times N G\right)$, while cGA requires only $(2 \times N G)$. As one can see from the results illustrated in Table 3, the difference between cGA for both the number of function evaluations and the percentage of the searched space until convergence in which cGA exhibits better 
performance than in the average of both cases. It is also worth noting that the number of function evaluations and the searched space are both decreases when the number of sample size increases.

Table 3: Average simulation results of Canonical GA and compact GA based on the number of function evaluations $F$ and the percentage of the searched space PSS

\begin{tabular}{|l|c|c|c|c|c|}
\hline \multirow{2}{*}{$\begin{array}{l}\text { Sample } \\
\text { Size (n) }\end{array}$} & $\boldsymbol{\theta}_{\mathbf{1}}$ & \multicolumn{2}{|c|}{ CGA } & \multicolumn{2}{c|}{$\boldsymbol{c G A}$} \\
\cline { 3 - 6 } & & $\boldsymbol{F}$ & $\boldsymbol{P S S}$ & $\boldsymbol{F}$ & $\boldsymbol{P S S}$ \\
\hline \multirow{4}{*}{25} & 0.1 & 3200 & 9.7656 & 124 & 0.3784 \\
\cline { 2 - 6 } & -0.1 & 4150 & 12.6647 & 130 & 0.3967 \\
\cline { 2 - 6 } & 0.4 & 3000 & 9.1552 & 116 & 0.3540 \\
\cline { 2 - 6 } & -0.4 & 3750 & 11.4440 & 124 & 0.3784 \\
\cline { 2 - 6 } & 0.7 & 3400 & 10.3759 & 138 & 0.4211 \\
\cline { 2 - 6 } & -0.7 & 3900 & 11.9018 & 140 & 0.4272 \\
\hline \multirow{5}{*}{50} & 0.1 & 3200 & 9.7656 & 122 & 0.3723 \\
\cline { 2 - 6 } & -0.1 & 3650 & 11.1389 & 126 & 0.3845 \\
\cline { 2 - 6 } & 0.4 & 2800 & 8.54492 & 100 & 0.3051 \\
\cline { 2 - 6 } & -0.4 & 3500 & 10.6811 & 120 & 0.3662 \\
\cline { 2 - 6 } & 0.7 & 3250 & 9.9182 & 128 & 0.3906 \\
\cline { 2 - 6 } & -0.7 & 3350 & 10.2233 & 120 & 0.3662 \\
\hline \multirow{5}{*}{100} & 0.1 & 2250 & 6.8664 & 96 & 0.2929 \\
\cline { 2 - 6 } & -0.1 & 3000 & 9.1552 & 106 & 0.3234 \\
\cline { 2 - 6 } & 0.4 & 2450 & 7.4768 & 98 & 0.2990 \\
\cline { 2 - 6 } & -0.4 & 3250 & 9.9182 & 116 & 0.3540 \\
\cline { 2 - 6 } & 0.7 & 2650 & 8.0871 & 102 & 0.3112 \\
\cline { 2 - 6 } & -0.7 & 2850 & 8.6975 & 110 & 0.3356 \\
\hline
\end{tabular}

From Figure 3 and 4 it is clear that the quality of solutions and convergence speed found by the cGA is better than the CGA. The results suggest that the cGA performs the best and the CGA performs the worst.

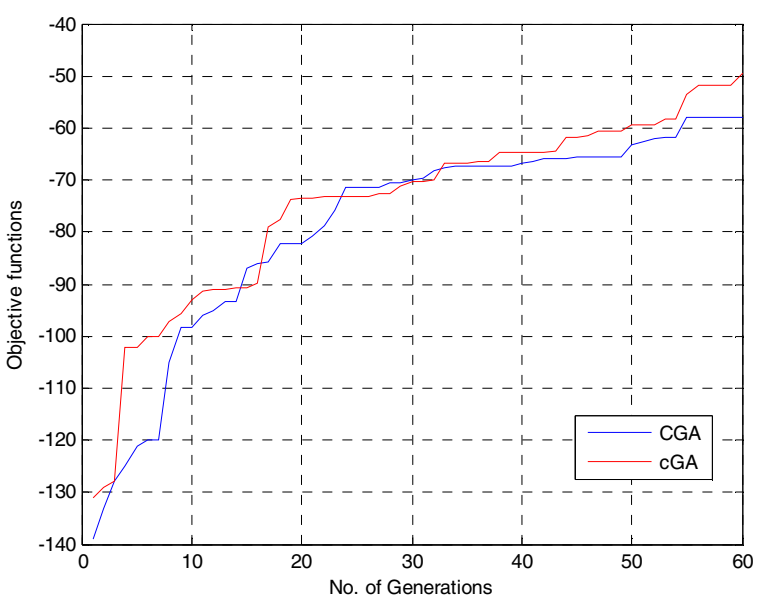

-a- Best Objective function of $\theta_{1}=0.1$

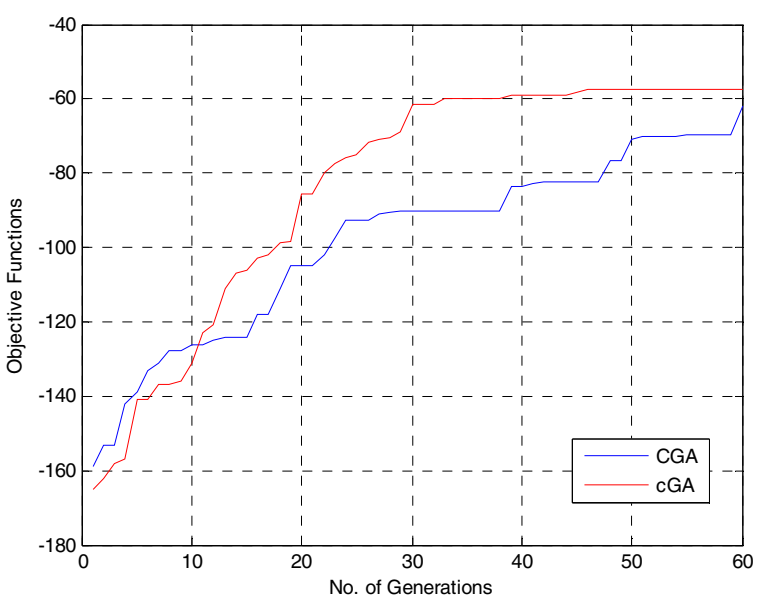

-b- Best Objective function of $\theta_{1}=0.4$

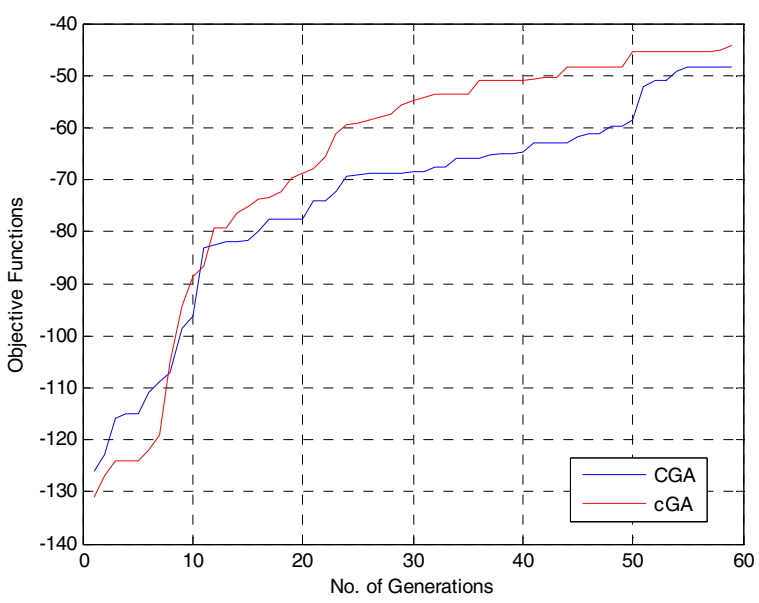

-c- Best Objective function of $\theta_{1}=0.7$

Figure 3. Results of best objective function (squared Error) of 60 generations over 5 Runs with $n=25$ 


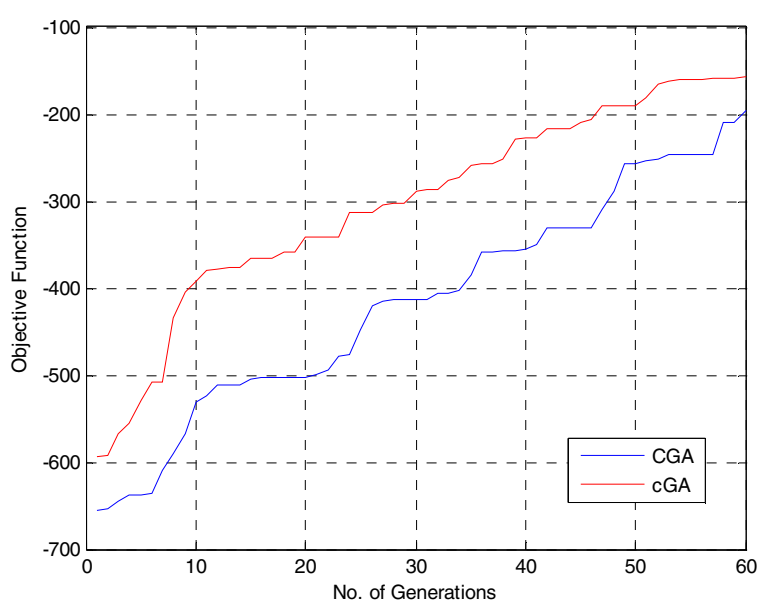

-d- Best Objective function of $\theta_{1}=-0.1$

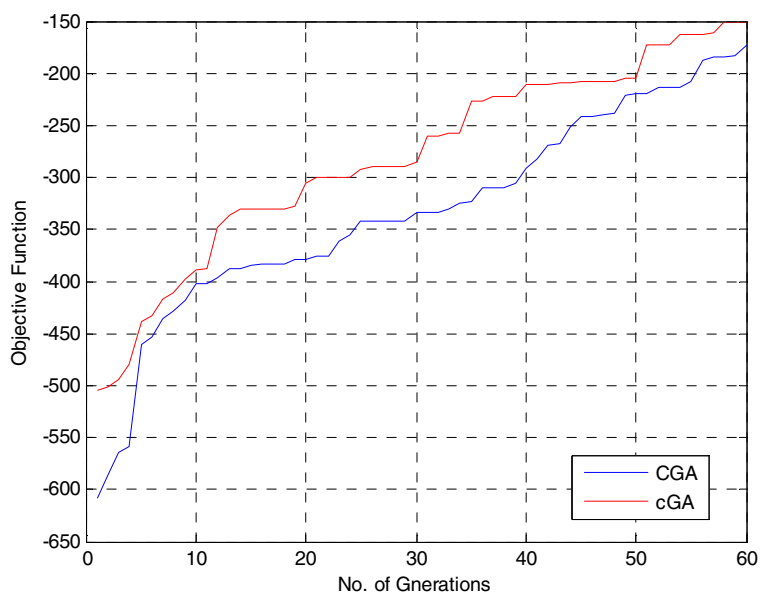

-e- Best Objective function of $\theta_{1}=-0.4$

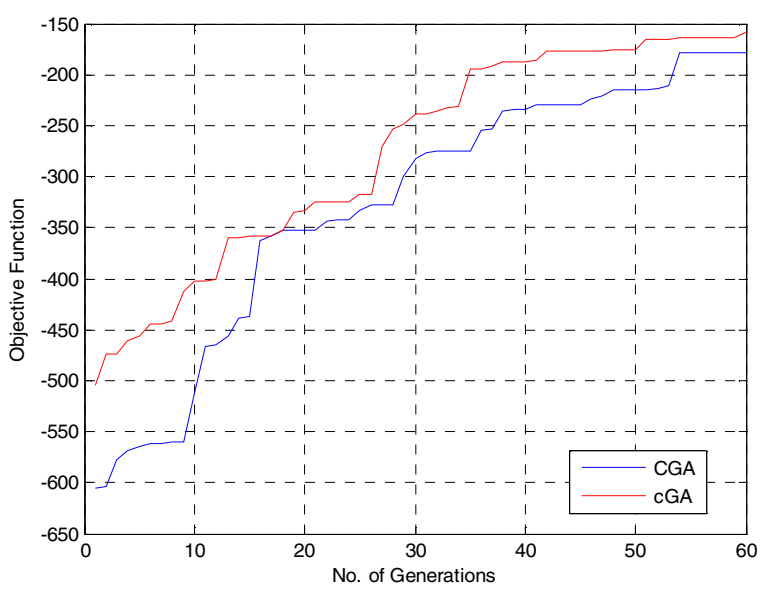

-f- Best Objective function of $\theta_{1}=-0.7$

Figure 4. Results of best objective function (Squared Error) of 60 generations over 5 Runs with $n=100$

\section{CONCLUSION}

In this paper, we investigate the performance of the compact Genetic Algorithm cGA for estimating the parameter of loglikelihood function $\ln \left(L\left(\theta, \sigma_{a}^{2}\right)\right)$ of first order moving average model $M A(1)$. Based on MSE, cGA provides effective results for three random samples with different sizes $(n=25,50,100)$ and $\theta$ is set to $(\bar{\mp} 0.1, \mp 0.4, \mp 0.7)$ in comparison with the CGA and Moment Method. Simulation results show that the cGA has a higher optimal precision or at least the same as that obtained from the CGA, at same time, the cGA needs minimum searched space percentage and less number of function evaluations than that of the CGA.

\section{REFERENCES}

[1] E. Goldberg David, "Genetic algorithms in search, optimization and machine learning," Reprinted with corrections from Goldberg (1989) Addison Wesley Longman Inc, 1993.

[2] P. Larranaga and J. A. Lozano, Estimation of distribution algorithms: A new tool for evolutionary computation vol. 2: Springer Netherlands, 2002.

[3] M. Pelikan, et al., "A survey of optimization by building and using probabilistic models," Computational optimization and applications, vol. 21, pp. 5-20, 2002.

[4] R. Rastegar and A. Hariri, "A step forward in studying the compact genetic algorithm," Evolutionary computation, vol. 14, pp. 277-289, 2006

[5] S. Baluja, "Population-based incremental learning. a method for integrating genetic search based function optimization and competitive learning," DTIC Document1994.

[6] S. Baluja and R. Caruana, "Removing the genetics from the standard genetic algorithm," in The International Conference on Machine Learning 1995, 1995, pp. 38-46.

[7] G. R. Harik, et al., "The compact genetic algorithm," Evolutionary Computation, IEEE Transactions on, vol. 3, pp. 287-297, 1999.

[8] W. W. S. Wei, Time series analysis: Addison-Wesley Redwood City, California, 1990.

[9] B. Hussain and R. Al-Dabbagh, "A canonical genetic algorithm for likelihood estimator of first order moving average model parameter," NEURAL NETWORK WORLD, vol. 17, pp. 271, 2007.

[10] B. A. H. Al-Sarray, "Variants of Hybrid Genetic Algorithms for Optimizing Likelihood ARMA Model Function and Many of Problems," Evolutionary Algorithms, pp. 219-246, 2011.

[11] S. Droste, "A rigorous analysis of the compact genetic algorithm for linear functions," Natural Computing, vol. 5, pp. 257-283, 2006.

[12] I. J. Myung, "Tutorial on maximum likelihood estimation," Journal of Mathematical Psychology, vol. 47, pp. 90-100, 2003. 
[13] G. E. P. Box and G. M. Jenkins, Time series analysis: forecasting and control: Prentice Hall PTR, 1994.

[14] D. F. Chrysoula, "Yule-Walker Estimation for the Moving-Average Model," International Journal of Stochastic Analysis, vol. 2011, 2011.

[15] ] C. Aporntewan and P. Chongstitvatana, "A hardware implementation of the Compact Genetic Algorithm," in Evolutionary Computation, 2001. Proceedings of the 2001 Congress on, 2001, pp. 624-629 vol. 1.

[16] M. Spyros, et al., "Forecasting: Methods and Applications," ed: New York, USA: John Wiley \& Sons Inc, 1997.

[17] S. Tsutsui, "Probabilistic model-building genetic algorithms in permutation representation domain using edge histogram," Parallel Problem Solving from Nature-PPSN VII, pp. 224-233, 2002. 